\title{
Investigation on Acoustic Properties of Thruster Chamber with Coaxial Injectors and Plenum Chamber
}

\author{
Dekun Gao, ${ }^{1}$ Jianxiu Qin $\mathbb{D}^{2},{ }^{2}$ and Huiqiang Zhang $\mathbb{D}^{1}$ \\ ${ }^{1}$ School of Aerospace Engineering, Tsinghua University, Beijing 100084, China \\ ${ }^{2}$ China Academy of Aerospace Aerodynamics, Beijing 100071, China \\ Correspondence should be addressed to Jianxiu Qin; qinjianxiu@hotmail.com \\ and Huiqiang Zhang; zhanghq@mail.tsinghua.edu.cn
}

Received 10 January 2020; Revised 15 April 2020; Accepted 18 May 2020; Published 25 September 2020

Academic Editor: Wei Lin

Copyright (c) 2020 Dekun Gao et al. This is an open access article distributed under the Creative Commons Attribution License, which permits unrestricted use, distribution, and reproduction in any medium, provided the original work is properly cited.

\begin{abstract}
Based on the URANS equation, a numerical simulation is carried out for acoustic properties of the thruster chamber with coaxial injectors and plenum chamber in a liquid rocket engine. Pressure oscillations with multiacoustic modes are successfully excited in the chamber by using the constant volume bomb method. FFT analysis is applied to obtain the acoustic properties of eigenfrequencies, power amplitudes, and damping rates for each excited acoustic mode. Compared with the acoustic properties in the model chamber with and without an injector as well as with and without the plenum chamber, it can be found that the injector with one open end and one half-open end still can work as a quarter-wave resonator. The power amplitudes of the acoustic mode can be suppressed significantly when its eigenfrequency is close to the tuning frequency of the injector, which is achieved by Cutting down the pressure Peak and Raising up the pressure Trough (CPRT). Compared with the acoustic properties in the model chamber with and without the plenum chamber, it can be found that $1 \mathrm{~L}$ acoustic pressure oscillation is inhibited completely by the plenum chamber and other acoustic pressure oscillations are also suppressed in a different extent. The injector and plenum chamber have a little effect on the eigenfrequencies and damping rate of each acoustic mode. For multimode pressure oscillation, it is better for tuning frequency of the injector closing to the lower eigenfrequency acoustic mode, which will be effective for suppression of these multiacoustic modes simultaneously.
\end{abstract}

\section{Introduction}

Gas-liquid coaxial injectors have been widely used in liquid rocket engines for their good properties on the mixing of fuel and oxygen, atomization of liquid propellant, and combustion stability of the thruster chamber. Many research works have been conducted on such injectors and related combustion chambers.

High-frequency combustion instability often occurs in liquid propellant engines and may be related to the geometry of the atomizing elements that are often used. Shear coaxial injector elements are particularly prone to such instability because the resonant acoustic frequencies of the LOX post are often close to the resonant frequencies of the combustion chamber [1]. A phenomenon on combustion instability in a subscale combustor for a LOX/methane rocket engine has been conducted by JAXA [2], in which the effects of five types of single shear coaxial injector on the combustion instability were observed. The injection-coupled response of a LOX post was predicted by Lin et al. based on the finite element model (FEM) [3], in which the observed instability was a result of the interaction of combustion chamber resonance frequencies with injector resonance frequencies. Injector-driven combustion instabilities in a hydrogen/oxygen rocket combustor were investigated by Groening et al. [4]. It was found that the oscillation of the heat release rate was related to the injector acoustics but was not corresponding with the combustion chamber pressure oscillations. Urbano et al. analyzed the combustion instability by using large eddy simulation in a liquid rocket engine with multiple coaxial injectors [5]; it was shown that the overall acoustic activity was strongly coupled with the injector. It can be found that the coaxial injector and its acoustic properties have an important effect on the combustion in the chamber of the 


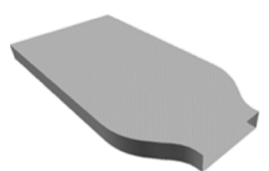

(a) Model A chamber without injectors and plenum chamber

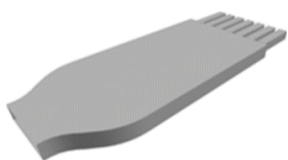

(b) Model B chamber with 7 injectors but without plenum chamber

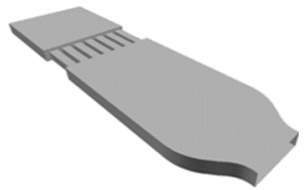

(c) Model C chamber with 7 injectors and plenum chamber

Figure 1: Configurations of three model chambers.

liquid rocket engine. So, the acoustic properties and decay rate of coaxial injectors have got much investigation.

Acoustic characteristics and acoustic damping induced by gas-liquid scheme coaxial injectors were studied by Zhou et al. $[6,7]$; it has been found that such injector can play a significant role in acoustic damping or absorption. Sohn et al. [8] examined combustion-stability characteristics of a coaxial injector by using a scaling method, in which similarity of acoustic, hydrodynamic, and combustion characteristics between a model test and an actual test was achieved. They [9] also studied acoustic fine-tuning of gas-liquid coaxial injectors by using linear acoustic analysis. Haksoon and Sohn [10] experimentally studied the gas-liquid coaxial injector as an acoustic resonator in the combustion chamber. Soller et al. [11] conducted a hot fire test in a thruster chamber with several coaxial swirl injectors under the condition of chamber pressures in the range of $4.0 \mathrm{MPa}$ to $8.5 \mathrm{MPa}$ and mixture ratios between 2.4 and 3.5, and the effects of the injector's parameters on the combustion instability were observed. Pomeroy et al. $[12,13]$ studied the response of combustion in a gas-centered swirl coaxial injector to high-amplitude, high-frequency, and transverse-mode pressure oscillation by using a high-speed camera. Kim and Heister [14] developed a homogeneous two-phase flow model to assess hydrodynamic instabilities of coaxial injectors, in which effects of the density ratio, gas-liquid velocity ratio, sheet thickness, channel length, and Reynolds number on such instabilities were discussed. The flame of a single coaxial injector was investigated by Zhukov and Feil [15]. And the spray characteristics of the gas-centered swirl coaxial injector were measured by Jeon et al. [16].

As shown above, many research works have been carried out to investigate the atomization, combustion, and acoustic properties of the gas-liquid coaxial injector. Indeed, such an injector is not like a typical acoustic resonator with one closed end, but it has one open end connected with the combustion chamber and one half-open end connected with the plenum chamber. Therefore, the acoustic properties of such an injector may be different from the typical acoustic resonator, and the plenum chamber may have a contribution to the decay of the pressure oscillation in the combustion chamber. But such contribution has never been evaluated till now. So, the acoustic properties of such an injector are investigated in this paper in a rocket engine combustor with a plenum chamber by using a numerical constant volume bomb method, in which a nonlinear pressure oscillation with multiacoustic modes is excited.

\section{Numerical Methodology}

2.1. Geometric Configurations. A model chamber sliced from a combustion chamber of a $100 \mathrm{kN}$ LOX/kerosene rocket engine is adopted in this paper for its less cost of computational time, in which seven gas-liquid coaxial injectors are included. In order to observe the effects of such injectors and the plenum chamber on the decay of pressure oscillation in the combustion chamber, three kinds of model chambers are presented in this paper. One is Model A, which is comprised of a combustion chamber without injectors and a plenum chamber. For Model B, seven gas-liquid coaxial injectors are equipped at the head of Model A. For Model $\mathrm{C}$, a plenum chamber is connected with those injectors in Model B. These three model chambers are shown in Figure 1. By comparing the decay processes of pressure oscillations in Models A and B, the effects of these injectors on the acoustic properties of the combustion chamber can be obtained, while by comparing the related processes in Model $\mathrm{B}$ and Model $\mathrm{C}$, the role of the plenum chamber on the decay of pressure oscillations can be identified.

The geometry sizes of the model chambers are shown in Figure 2. The model chamber is a center slice of a fullscaled chamber, and it keeps the same geometrical parameters in transverse ( $x$ direction) and longitudinal ( $z$ direction) dimensions as a full-scaled chamber. The lengths from the injector plate to the nozzle entrance and throat are $130 \mathrm{~mm}$ and $180 \mathrm{~mm}$, respectively. The transverse sizes of the model chamber and nozzle throat are $88 \mathrm{~mm}$ and $45.2 \mathrm{~mm}$, respectively. The thickness (spanwise size) of this slice chamber is $12 \mathrm{~mm}$. The transverse and spanwise ( $y$ direction) sizes of these injectors are $10 \mathrm{~mm}$, while their length is set as 22, 32, $44,62,78$, and $100 \mathrm{~mm}$ for different cases. The length and width of the plenum chamber are $100 \mathrm{~mm}$ and $88 \mathrm{~mm}$, respectively. Compared with transverse size of the injector with $10 \mathrm{~mm}$, there is a contraction with $5 \mathrm{~mm}$ at the inlet of the injector, which is shown in Figures 1 and 2. So, the injector's outlet is an open end, while its inlet is a half-open end; it is corresponding to that in engineering. 


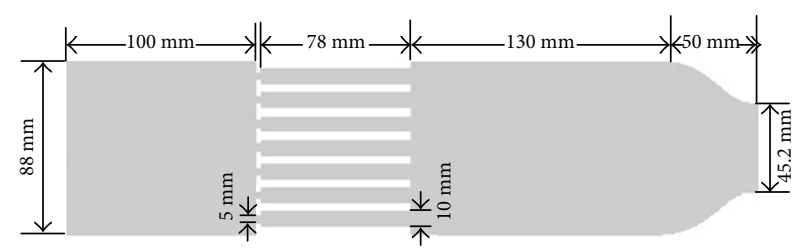

(a) Front view

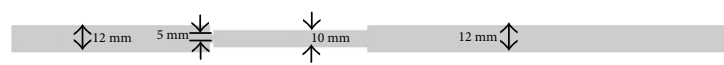

(b) Top view

Figure 2: The geometry size of the thrust chamber, injector, and plenum chamber.

2.2. Governing Equation. Investigation on the acoustic performance of the combustion chamber under cold condition in normal temperature and pressure environment is generally adopted in engineering to evaluate the chamber's eigenfrequencies and decay rates [17]. So, the model chambers are initially filled with quiescent air with a pressure of $0.1 \mathrm{MPa}$ and a temperature of $300 \mathrm{~K}$. Instead of loudspeakers or bombs in engineering used as a stimulated source, a pressure pulse is imposed on a determined region in the model chambers in present works. The stimulated turbulent flow and pressure oscillations in these chambers are simulated by the URANS method and compared with each other, in which the turbulent is described by the $k-\varepsilon$ model. The governing equations for turbulent flow in the model chambers can be written as follows:

$$
\begin{gathered}
\frac{\partial \rho}{\partial t}+\frac{\partial \rho u_{j}}{\partial x_{j}}=0, \\
\frac{\partial\left(\rho u_{i}\right)}{\partial t}+\frac{\partial\left(\rho u_{i} u_{j}\right)}{\partial x_{j}}=-\frac{\partial p}{\partial x_{j}}-\frac{\partial}{\partial x_{j}}\left(\frac{2}{3} \rho k\right)+\frac{\partial \tau_{i j}}{\partial x_{j}}, \\
\frac{\partial(\rho e)}{\partial t}+\frac{\partial\left(\rho u_{j} e\right)}{x_{j}}=-p \frac{\partial u_{j}}{\partial x_{j}}-\frac{\partial}{\partial x_{j}}\left(-K \frac{\partial T}{\partial x_{j}}-\rho D h\right)+\rho \varepsilon \\
\frac{\partial(\rho k)}{\partial t}+\frac{\partial\left(\rho u_{j} k\right)}{\partial x_{j}}=-\frac{2}{3} \rho k \frac{\partial u_{j}}{\partial x_{j}}+\tau_{i j} \frac{\partial u_{i}}{\partial u_{j}}+\frac{\partial}{\partial x_{j}}\left(\frac{\mu_{e}}{\sigma_{k}} \frac{\partial k}{\partial x_{j}}\right)-\rho \varepsilon, \\
+\frac{\partial(\rho \varepsilon)}{\partial t}+\frac{\partial\left(\rho u_{j} \varepsilon\right)}{\partial x_{j}}=-\left(\frac{2}{3} C_{\varepsilon 1}-C_{\varepsilon 3}\right) \rho \varepsilon \frac{\partial u_{j}}{\partial x_{j}}+\frac{\partial}{\partial x_{j}}\left(\frac{\mu_{e}}{\sigma_{\varepsilon}} \frac{\partial \varepsilon}{\partial x_{j}}\right) \\
\left.C_{\varepsilon 1} \tau_{i j} \frac{\partial u_{i}}{\partial x_{j}}-C_{\varepsilon 2} \rho \varepsilon\right),
\end{gathered}
$$

where $\rho, p, u_{j}, e, T, h, k$, and $\varepsilon$ represent the density, pressure, velocity in the $j$ th direction, total energy, temperature, enthalpy, turbulent kinetic energy, and its dissipation rate, respectively. $D$ and $K$ are diffusion coefficient and heat conduction coefficient, respectively. For effective viscosity $\mu_{e}$ and viscous stress tensor $\tau_{i j}$, we have

$$
\mu_{e}=\mu+\mu_{t}
$$

TABLE 1: Turbulence model coefficients.

\begin{tabular}{lllllll}
\hline Parameter & $\mu$ & $C_{\varepsilon 1}$ & $C_{\varepsilon 2}$ & $C_{\varepsilon 3}$ & $\sigma_{k}$ & $\sigma_{\varepsilon}$ \\
\hline Value & 0.09 & 1.44 & 1.92 & -1.0 & 1.0 & 1.3 \\
\hline
\end{tabular}

$$
\tau_{i j}=\mu_{e}\left(\frac{\partial u_{i}}{\partial x_{j}}+\frac{\partial u_{j}}{\partial x_{i}}\right)-\frac{2}{3} \mu_{e} \frac{\partial u_{j}}{\partial x_{j}} \delta_{i j}
$$

where

$$
\mu_{t}=\frac{\rho C_{\mu} k^{2}}{\varepsilon}
$$

The turbulent model coefficients are shown in detail in Table 1 , in which the standard coefficients are adopted in the present works.

2.3. Numerical Algorithm and Boundary Conditions. The semi-implicit method for pressure linked equations is adopted to solve the pressure field. And the forward-Euler difference method is employed for temporal difference. The finite volume method is adopted for spatial difference, in which the diffusion terms and convection terms are discretized by a second-order central difference scheme and second-order upwind scheme, respectively.

The injector face of the combustion chamber, as well as side walls of thruster chambers, injector, and plenum chamber, is set as wall boundary. The adiabatic condition and turbulent law-of-the-wall condition are adopted to wall boundary. In order to avoid setting as a closed end for the upstream boundary of injectors for Model B chamber and for the upstream boundary of the plenum chamber in Model $\mathrm{C}$ chamber, a small velocity and a given pressure are set at these upstream boundaries. The chamber exit is set as an outlet boundary, in which the pressure at the exit is set as ambient pressure and the velocity is set equal to that of the logical inside neighbor vertex. It means that the partial derivative in the streamwise direction is set to zero at the outlet. The boundary condition in the nozzle throat for the cold case is different from the real hot case. There is no reflection of the pressure wave at the throat in the real case, but in our case, the pressure wave may be reflected at the exit where the pressure is set as the ambient pressure, which may influence the longitudinal acoustic modes. However, the longitudinal acoustic modes are mainly dominated by the wall of the convergence section of the nozzle which is the same for the 
present case and real case. So, the above boundary condition for the outlet is acceptable.

The grid scales are $2 \mathrm{~mm}, 1 \mathrm{~mm}$, and $2 \mathrm{~mm}$ in the $x, y$, and $z$ directions, respectively. The time step is set as $5 \times 10^{-7} \mathrm{~s}$, but it is $1 \times 10^{-8} \mathrm{~s}$ during the period of the constant volume bomb imposed on the steady flow in the chamber.

The initial condition is that the model chambers are filled with air at $1 \mathrm{~atm}$ and $300 \mathrm{~K}$. As shown in Figure 3, a region $\Omega$ (colored by red) is selected to impose the constant volume bomb; it is a $6 \mathrm{~mm} \times 6 \mathrm{~mm} \times 6 \mathrm{~mm}$ cube with the central coordinate $(37 \mathrm{~mm}, 0$, and $7 \mathrm{~mm}$ ). It is $4 \mathrm{~mm}$ far from the injection panel and the upper wall of the chamber. At this region, the initial pressure, temperature, and density are detonated as $P_{0}, T_{0}$, and $\rho_{0}$, respectively. A gas with pressure $\gamma P_{0}$ , temperature $\alpha T_{0}$, and density $\beta \rho_{0}$ is added into this region $\Omega$ from the initial time $t_{0}$ to time $t_{0}+\Delta t$, which has the same components with the original gas in the imposed region $\Omega$. Supposing that the imposed gas and the original gas are mixed quickly through a constant volume process, the pressure $P(x, y, z, t)$, temperature $T(x, y, z, t)$, and density $\rho$ $(x, y, z, t)$ at the imposed region $\Omega$ after imposing are thereby obtained as follows:

$$
\begin{gathered}
P(x, y, z, t)=(1+\gamma) P_{0}, \\
\rho(x, y, z, t)=(1+\beta) \rho_{0}, \\
T(x, y, z, t)=\left(\frac{1+\gamma}{1+\beta}\right) T_{0}, \\
\gamma=\alpha \beta \\
(x, y, z) \in \Omega, \\
t_{0} \leq t \leq t_{0}+\Delta t .
\end{gathered}
$$

A pressure pulse is then achieved as a stimulated source by this method. $\alpha, \gamma$, and $\beta$ are model coefficients. The temperature of the imposed region usually keeps the same before and after the imposing process; thus, we have $\alpha=1$ and $\gamma=\beta$ . Only one model coefficient $\gamma$ is required to be predetermined in this model, by which the peak value of such pressure pulse can be selected. In this paper, $\gamma$ and $\Delta t$ are taken as 19 and $10^{-6} \mathrm{~s}$, and then, a pressure pulse as 20 times higher than the initial pressure is achieved in the model chambers with a period of $10^{-6} \mathrm{~s}$ to induce a pressure oscillation. For all model chambers, the location and intensity of the pressure pulse are the same. After the constant volume bomb is imposed in the chamber, several positions near the chamber wall and near the injector face are selected to observe the pressure oscillation. For the sliced chamber adopted in this paper, transverse ( $x$ direction) and longitudinal ( $z$ direction) acoustic modes recorded in these observation points are almost the same. In order to save space, the results for only one observation point are shown in the paper. This observation point locates at the opposite side of the bomb; its pressure is applied to study the acoustic properties of the chamber.

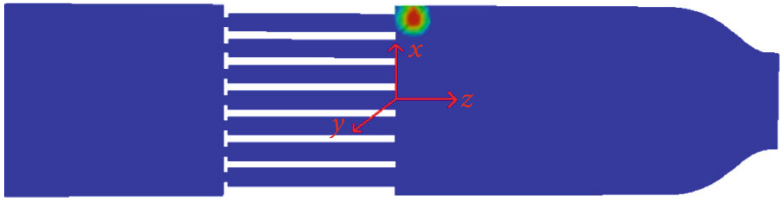

FIgURe 3: The imposed region of the constant volume bomb in the model chamber.

2.4. Quantification of Acoustic Damping Capacity. Halfpower bandwidth $\Delta f_{n}$ is adopted to evaluate the acoustic damping capacity of each model chamber in this paper. Pressure oscillations with large amplitude are achieved in the model chamber by using the above numerical constant volume bomb. FFT analysis is then carried out, and some power peaks in the frequency region can be obtained. For the $n_{\text {th }}$ peak with power amplitude $P_{n \text {,peak }}$ at corresponding frequency $f_{n}$, half-power bandwidth $\Delta f_{n}$ is calculated as the following:

$$
\Delta f_{n}=\left(f_{n, 2}-f_{n, 1}\right)
$$

where $f_{n, 2}$ and $f_{n, 1}$ are the frequencies at which the power amplitude of the pressure oscillation corresponds to $P_{n \text {,peak }} /$ $\sqrt{2}$ with $f_{n, 2}>f_{n, 1}$. Once the half-power bandwidth has been determined, the damping rate $\alpha_{n}$ of the model chamber for this acoustic mode with the peak frequency $f_{n}$ can be figured out by the following relation:

$$
\alpha_{n}=\pi \Delta f_{n}
$$

Furthermore, the nondimensional damping factor $\eta_{n}$ for a peak frequency $f_{n}$ is defined by the half-power bandwidth method as follows:

$$
\eta_{n}=\frac{\alpha_{n}}{\pi f_{n}}=\frac{\Delta f_{n}}{f_{n}} .
$$

As shown in this equation, the bigger the half-power bandwidth is, the higher the damping rate is, which means that pressure oscillations of the corresponding acoustic mode decay faster. Hence, half-power bandwidth, which is dimensionless by eigenfrequency, is applied as a quantification parameter of the damping factor of the chamber in this paper.

\section{Results and Discussion}

The numerical method applied in this paper for acoustic properties of a combustion chamber has been validated for a small thrust liquid rocket engine chamber by experimental results [18]. So, the validation of the numerical method is no longer done again in this paper. On the other hand, there is a lack of experimental data for the cases in this paper.

3.1. Grid Convergence Study. Acoustic performances for three model chambers, including their acoustic-mode eigenfrequencies and damping capacities, are mainly considered in 


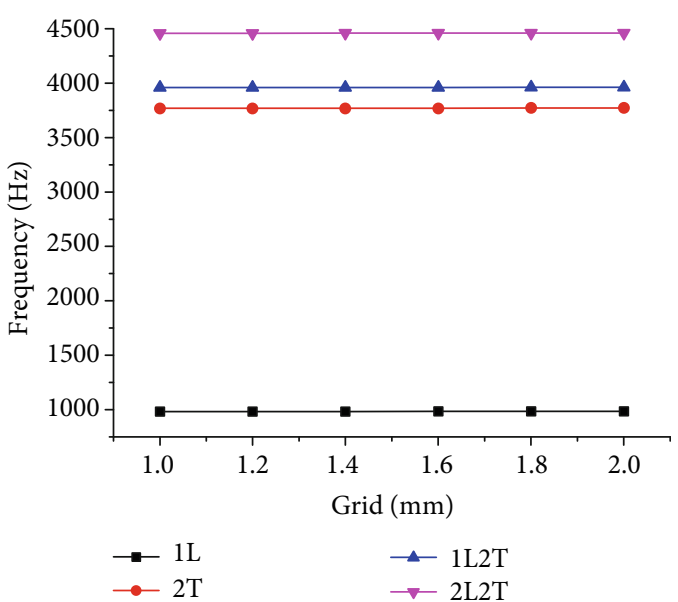

(a) Eigenfrequency

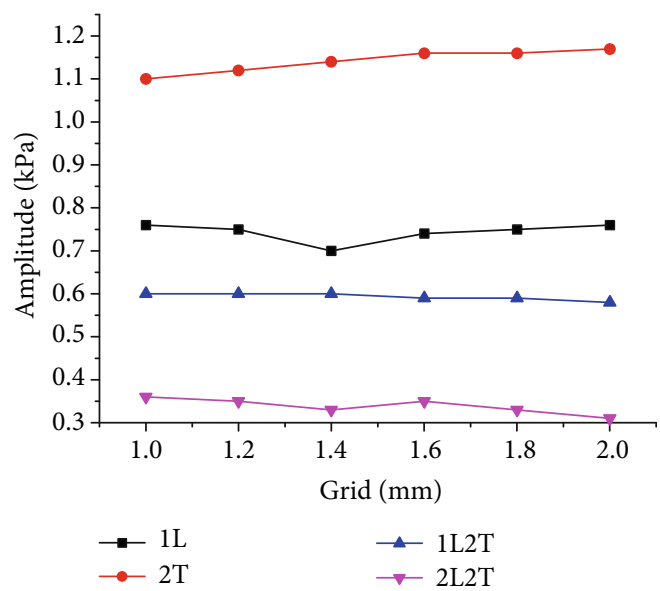

(b) Amplitude

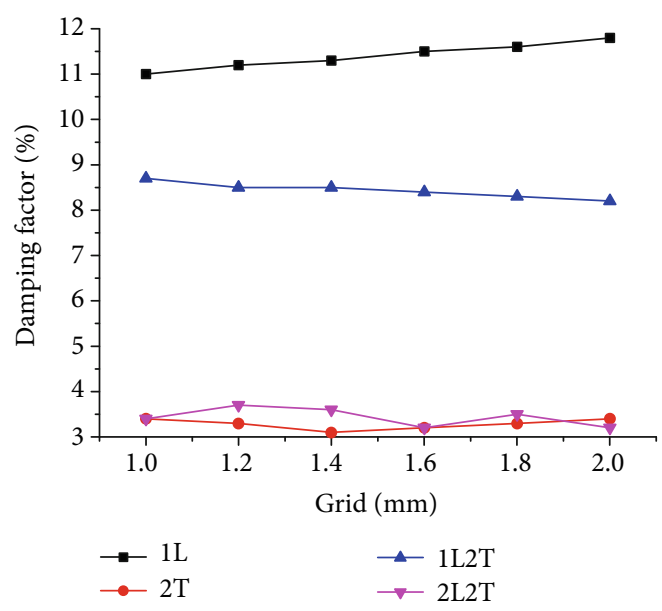

(c) Damping factor

FIgURE 4: Acoustic performances of the chamber with different grid sizes.

this paper. So, grid convergences are examined based on these acoustic performances. Grid sizes varied from $1 \mathrm{~mm}$ to $2 \mathrm{~mm}$ in the $x$ and $z$ directions are employed in grid convergence study. The eigenfrequencies and damping factors of acoustic modes for the Model A chamber are shown in Figure 4. For grid sizes varied from $1 \mathrm{~mm}$ to $2 \mathrm{~mm}$, the identified acoustic modes are the same, and the frequencies, amplitudes, and damping factors of these acoustic modes do not have any significant difference. In order to reduce the computation cost, $2.0 \mathrm{~mm}$ grid size is enough to predict the acoustic performances of the chamber used in this paper.

3.2. Acoustic Properties of Model A Chamber. The eigenfrequencies of acoustic modes of the Model A chamber are obtained by theoretical calculation, in which the combustion chamber with the nozzle convergence section is treated theoretically as a closed cylindrical chamber. The eigenfrequencies for a cylindrical chamber closed at both sides can be calculated with the following:
TABLE 2: Theoretical eigenfrequencies of acoustic modes in the model chamber.

\begin{tabular}{lrcc}
\hline Longitudinal (L) & 0 & Transverse (T) & \multicolumn{1}{c}{1} \\
\hline 0 & - & $1875 \mathrm{~Hz}$ & $3750 \mathrm{~Hz}$ \\
1 & $1064 \mathrm{~Hz}$ & $2155 \mathrm{~Hz}$ & $3898 \mathrm{~Hz}$ \\
2 & $2128 \mathrm{~Hz}$ & $2836 \mathrm{~Hz}$ & $4311 \mathrm{~Hz}$ \\
\hline & $f_{l m n}=\frac{c}{2 \pi} \sqrt{\frac{\lambda_{m n}^{2}}{R_{c}^{2}}+\frac{l^{2} \pi^{2}}{L_{c}^{2}}}$, & &
\end{tabular}

where $\lambda_{m n}$ is the transverse eigenvalue [17]. $c$ is the speed of sound and taken as $340 \mathrm{~m} / \mathrm{s}$ in the present cases. For combustion chambers as shown in Figure 1, $L_{c}$ is the effective acoustic length, which is taken as the sum of chamber length and one-half of the converging nozzle length.

Based on Equation (17), the theoretical eigenfrequencies of acoustic modes of the Model A chamber are shown in Table 2 . The $1 \mathrm{~L}$ acoustic mode has the lowest eigenfrequency. 


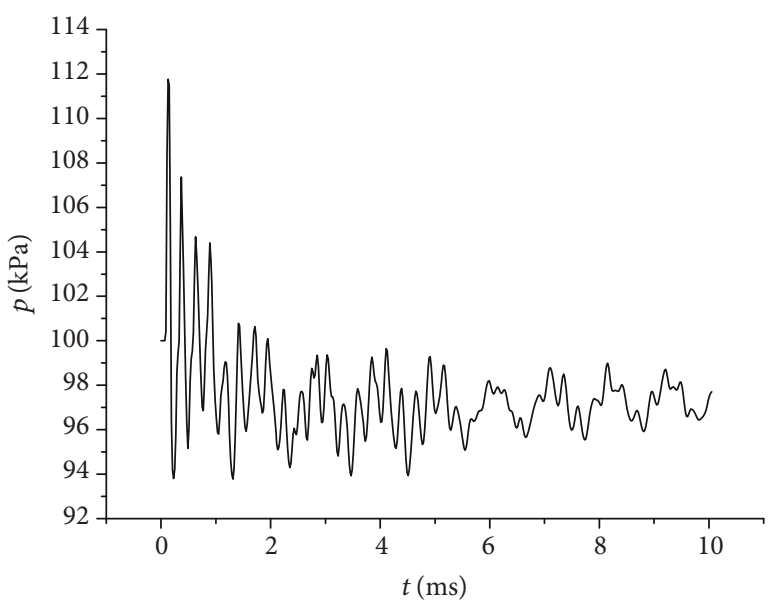

(a) Pressure oscillations

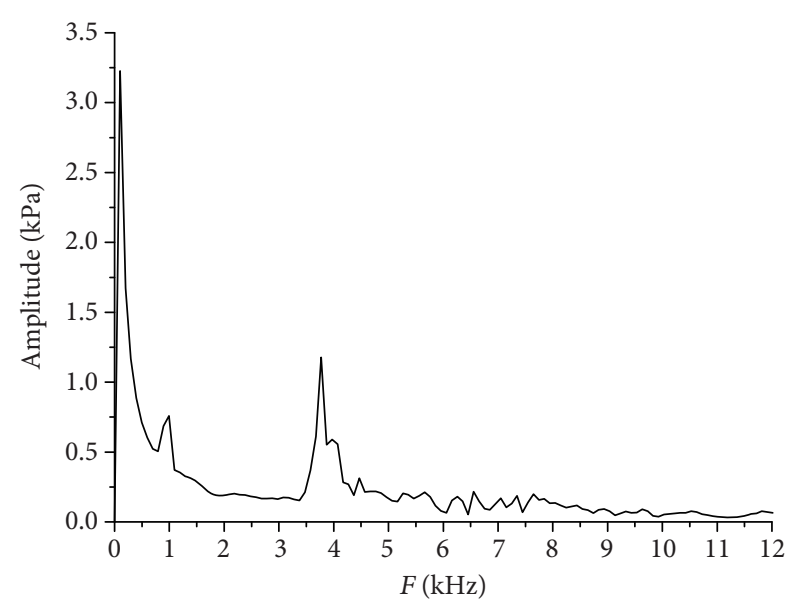

(b) FFT analysis

FIgURe 5: Pressure oscillations and their FFT analysis at the observation point for Model A chamber.

The $1 \mathrm{~T}$ and $2 \mathrm{~T}$ acoustic modes are very important for engineering; their eigenfrequencies are $1875 \mathrm{~Hz}$ and $3750 \mathrm{~Hz}$. These eigenfrequencies are applied to identify the acoustic modes excited by the numerical constant volume bomb in the model chambers in the following analysis.

After imposing a constant volume bomb in the Model A chamber, the pressure oscillations at the observation point are shown in Figure 5(a), and their corresponding FFT analysis is shown in Figure 5(b). Four peak frequencies are observed as $985 \mathrm{~Hz}, 3771 \mathrm{~Hz}, 3961 \mathrm{~Hz}$, and $4461 \mathrm{~Hz}$. Compared with the theoretical eigenfrequencies of acoustic modes for this chamber as shown in Table 2, $985 \mathrm{~Hz}, 3771 \mathrm{~Hz}, 3961 \mathrm{~Hz}$, and $4461 \mathrm{~Hz}$ of these four peak frequencies are identified as the first longitudinal (1L) acoustic mode, second transverse (2T) acoustic mode, $1 \mathrm{~L} 2 \mathrm{~T}$ acoustic mode, and 2L2T acoustic mode, respectively. Furthermore, the eigenfrequencies, power amplitudes, and damping rate of these four acoustic modes are therefore obtained and shown in Table 3. For peak frequency $3961 \mathrm{~Hz}$, though it is identified as the 1L2T acoustic mode, it is difficult to determine its half-power bandwidth for its peak is not isolated with a small peak value. Only frequency and amplitude are given for this peak, and its damping factor is not available.

Comparing the power amplitudes of these four acoustic modes, the $1 \mathrm{~L}$ and $2 \mathrm{~T}$ modes are the acoustic modes that can be excited most easily in this chamber for they have large power amplitudes. The other two acoustic modes of $1 \mathrm{~L} 2 \mathrm{~T}$ and $2 \mathrm{~L} 2 \mathrm{~T}$ may appear due to interaction of $1 \mathrm{~L}$ and $2 \mathrm{~T}$ acoustic modes, so they have small power amplitudes. Comparing the damping rate of these four acoustic modes, the $1 \mathrm{~L}$ mode is the acoustic mode that can be decayed most rapidly for they have a large damping rate, while $2 \mathrm{~T}$ and 2L2T modes are acoustic modes that can be decayed most difficulty for their small damping rates. The damping rate of $1 \mathrm{~L} 2 \mathrm{~T}$ is between those of $1 \mathrm{~L}$ acoustic mode and $2 \mathrm{~T}$ acoustic mode. Though the eigenfrequency of $1 \mathrm{~L}$ is smaller than those of the other three acoustic modes, it has the largest damping rate due to the open downstream condi-
TABLE 3: Eigenfrequency, power amplitude, and damping rate of acoustic modes in the Model A chamber.

\begin{tabular}{lcc}
\hline Acoustic modes & Properties & Values \\
\hline \multirow{3}{*}{ 1L acoustic mode } & Eigenfrequency $(\mathrm{Hz})$ & 985 \\
& Amplitude $(\mathrm{kPa})$ & 0.76 \\
& Damping factor & $11.8 \%$ \\
\hline \multirow{2}{*}{ 2T acoustic mode } & Eigenfrequency $(\mathrm{Hz})$ & 3771 \\
& Amplitude $(\mathrm{kPa})$ & 1.17 \\
& Damping factor & $3.4 \%$ \\
\hline \multirow{2}{*}{ L2T acoustic mode } & Eigenfrequency $(\mathrm{Hz})$ & 3961 \\
& Amplitude $(\mathrm{kPa})$ & 0.58 \\
& Damping factor & - \\
\hline \multirow{2}{*}{ 2L2T acoustic mode } & Eigenfrequency $(\mathrm{Hz})$ & 4461 \\
& Amplitude $(\mathrm{kPa})$ & 0.31 \\
& Damping factor & $3.2 \%$ \\
\hline
\end{tabular}

tions. So, the present results are reasonable. In general, based on theoretical acoustic modes and spatiotemporal evolution of instantaneous pressure distribution in the chamber, the peak frequencies obtained by the present simulation can be successfully identified. It can be concluded that multimode acoustic pressure oscillations are stimulated by the present numerical constant volume bomb model, and acoustic properties like eigenfrequencies, power amplitudes, and damping rate are given quantitively in the present work.

3.3. Acoustic Properties of Model B Chamber. The gas injector in the coaxial injector is not like a typical quarter-wave resonator for it has two open ends. However, the end of this injector at the plenum side is usually a half-open end for there is a contraction. So, the coaxial injector is usually considered as a quarter-wave resonator in engineering, which is widely used to suppress combustion instability in a liquid rocket engine. The tuning frequencies $f_{0}$ of such an injector are determined 
TABLE 4: Eigenfrequency, power amplitude, and damping rate of acoustic modes in the Model B chamber.

\begin{tabular}{|c|c|c|c|c|c|c|c|c|}
\hline & \multicolumn{7}{|c|}{ Length of the injector $(\mathrm{cm})$} \\
\hline & & 0 & 2.2 & 3.2 & 4.4 & 6.2 & 7.8 & 10 \\
\hline \multicolumn{2}{|c|}{ Resonator's tuning frequency $\left(f_{0}, \mathrm{~Hz}\right)$} & - & 3750 & 2578 & 1875 & 1330 & 1057 & 825 \\
\hline \multirow{3}{*}{$1 \mathrm{~L}$ acoustic mode } & Eigenfrequency $(\mathrm{Hz})$ & 985 & 944 & 853 & - & - & - & 1126 \\
\hline & Amplitude (kPa) & 0.76 & 1.1 & 1.1 & - & - & - & 0.58 \\
\hline & Damping factor & $11.8 \%$ & $11.2 \%$ & $17.3 \%$ & - & - & - & $11.5 \%$ \\
\hline \multirow{3}{*}{$2 \mathrm{~T}$ acoustic mode } & Eigenfrequency $(\mathrm{Hz})$ & 3771 & 3777 & 3791 & 3798 & 3798 & 3842 & 3567 \\
\hline & Amplitude (kPa) & 1.17 & 0.72 & 1.1 & 1.15 & 0.31 & 0.57 & 1.18 \\
\hline & Damping factor & $3.4 \%$ & $3.5 \%$ & $2.5 \%$ & $2.1 \%$ & - & $3.5 \%$ & $2.1 \%$ \\
\hline \multirow{3}{*}{$1 \mathrm{~L} 2 \mathrm{~T}$ acoustic mode } & Eigenfrequency $(\mathrm{Hz})$ & 3961 & 4155 & 4076 & 3988 & 4178 & 4049 & 4131 \\
\hline & Amplitude (kPa) & 0.58 & 1.1 & 1.65 & 0.65 & 0.8 & 0.89 & 0.61 \\
\hline & Damping factor & $8.2 \%$ & $2.4 \%$ & $2.4 \%$ & $3.2 \%$ & $4.3 \%$ & $2.0 \%$ & $3.4 \%$ \\
\hline \multirow{3}{*}{ 2L2T acoustic mode } & Eigenfrequency $(\mathrm{Hz})$ & 4461 & 4626 & - & 4368 & 4558 & - & 4507 \\
\hline & Amplitude (kPa) & 0.31 & 0.8 & - & 0.33 & 0.49 & - & 0.44 \\
\hline & Damping factor & $3.2 \%$ & $3.7 \%$ & - & $1.9 \%$ & $4.6 \%$ & - & $2.0 \%$ \\
\hline \multirow{3}{*}{ Unidentified frequency peak } & Eigenfrequency $(\mathrm{Hz})$ & - & - & - & - & 3323 & - & - \\
\hline & Amplitude (kPa) & - & - & - & - & 0.59 & - & - \\
\hline & Damping factor & - & - & - & - & $5.3 \%$ & - & - \\
\hline
\end{tabular}

by its length $L_{\text {inj. }}$. As the following equation,

$$
f_{0}=\frac{c}{4\left(L_{\text {inj }}+\Delta L\right)},
$$

where $f_{0}$ denotes tuning frequency of the injector, $c$ is the sound speed of gas in the injector, and $\Delta L$ is the length correction factor, which is neglected in this paper.

In order to cover the eigenfrequencies of acoustic modes shown in Table 2, which are possible to appear in the model chamber, a series of injector length are selected in this paper. The tuning frequencies for different length injectors are shown in Table 4, which vary from $825 \mathrm{~Hz}$ to $3750 \mathrm{~Hz}$. The eigenfrequencies of $1 \mathrm{~L}$ and $2 \mathrm{~T}$ acoustic modes in the model chamber are exactly covered.

After imposing a constant volume bomb in the Model B chamber, FFT analysis of pressure oscillations at the observation point for cases with different injector lengths is shown in Figure 6. For the Model B chamber, it is difficult to obtain its eigenfrequencies of acoustic modes through theoretical calculation, so the peak frequencies that appeared in Figure 6 are also identified by the theoretical eigenfrequencies of acoustic modes for the Model A chamber. There are four peak frequencies among these results for the Model B chamber; they are located between $853 \mathrm{~Hz}$ and $1126 \mathrm{~Hz}, 3323 \mathrm{~Hz}$ and $3777 \mathrm{~Hz}, 4049 \mathrm{~Hz}$ and $4155 \mathrm{~Hz}$, and $4368 \mathrm{~Hz}$ and $4626 \mathrm{~Hz}$, respectively. And based on the theoretical eigenfrequencies of acoustic modes for the Model A chamber, they are therefore identified as eigenfrequencies of $1 \mathrm{~L}, 2 \mathrm{~T}, 1 \mathrm{~L} 2 \mathrm{~T}$, and 2L2T acoustic modes, respectively. So, the excited acoustic modes in the Model B chamber by the same numerical constant volume bomb are still those of $1 \mathrm{~L}, 2 \mathrm{~T}, 1 \mathrm{~L} 2 \mathrm{~T}$, and 2L2T modes. Compared with those in the Model A chamber, there is no new acoustic mode that appeared in the Model B chamber. So, the acoustic modes excited by the same stimulated source do not change for the case with or without injectors. It mainly depends on the chamber configuration for the observed acoustic-mode pressure oscillation propagation in the chamber.

The eigenfrequencies, power amplitudes, and damping rate of $1 \mathrm{~L}, 2 \mathrm{~T}, 1 \mathrm{~L} 2 \mathrm{~T}$, and $2 \mathrm{~L} 2 \mathrm{~T}$ acoustic modes observed through Figure 6 in the Model B chamber are listed in Table 4. In order to compare with those in the Model A chamber directly, the results for the Model A chamber are also listed in Table 4 and marked by zero length of the injector. The eigenfrequencies and damping rate of each acoustic mode for the Model B chamber are close to those of the corresponding acoustic mode for the Model A chamber. So, with or without an injector does not have a significant effect on the eigenfrequency of the acoustic mode. And the extra decay due to propagation of the pressure wave in the injector is not the main way to suppress the pressure oscillations.

However, injectors have a great effect on the power amplitudes of the acoustic modes which even decrease to zero for some cases. For the case of injector length with $2.2 \mathrm{~cm}$, the tuning frequency of such injector is close to the eigenfrequency of the $2 \mathrm{~T}$ acoustic mode. Compared with the amplitude of the $2 \mathrm{~T}$ acoustic mode in the Model A chamber, it decreases obviously for this case with injectors. However, the pressure oscillation of $1 \mathrm{~L}$ acoustic mode is enhanced due to the redistribution of oscillation energy among these acoustic modes. For the case of injector length with $3.2 \mathrm{~cm}$, the tuning frequency is not close to either eigenfrequencies of $1 \mathrm{~L}$ and $2 \mathrm{~T}$ acoustic modes, so the pressure oscillations of these two acoustic modes are not restrained anymore. But the pressure oscillation of the 2L2T acoustic mode disappears for this case. The tuning frequency of the 


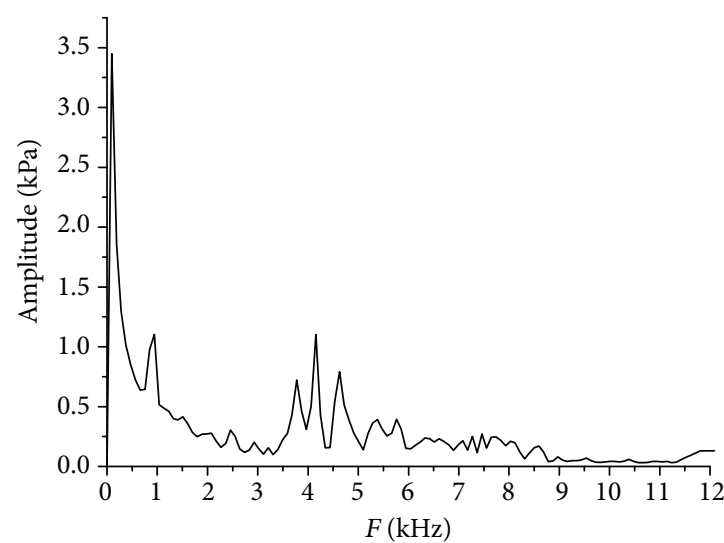

(a) $2.2 \mathrm{~cm}$

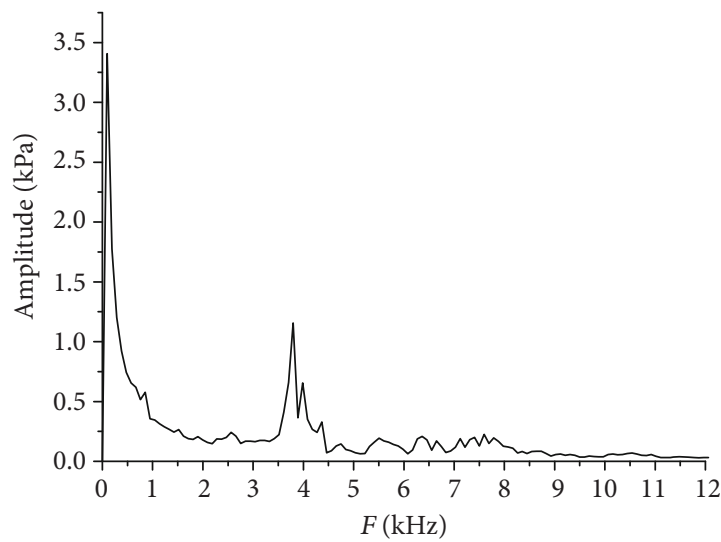

(c) $4.4 \mathrm{~cm}$

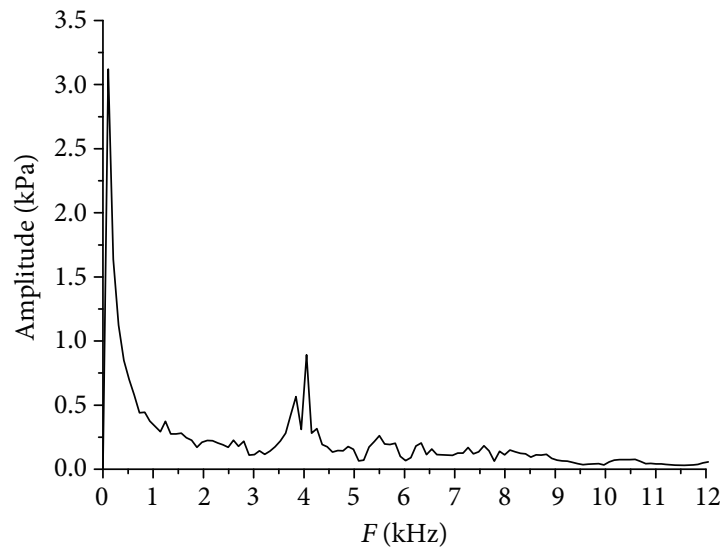

(e) $7.8 \mathrm{~cm}$

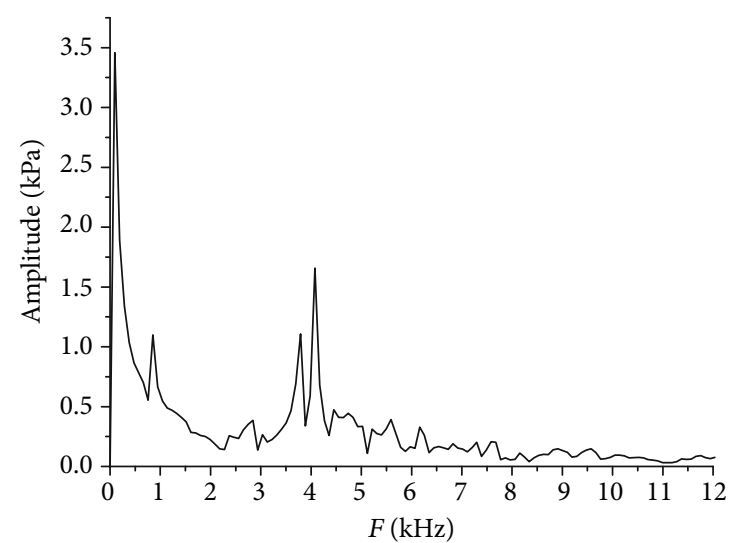

(b) $3.2 \mathrm{~cm}$

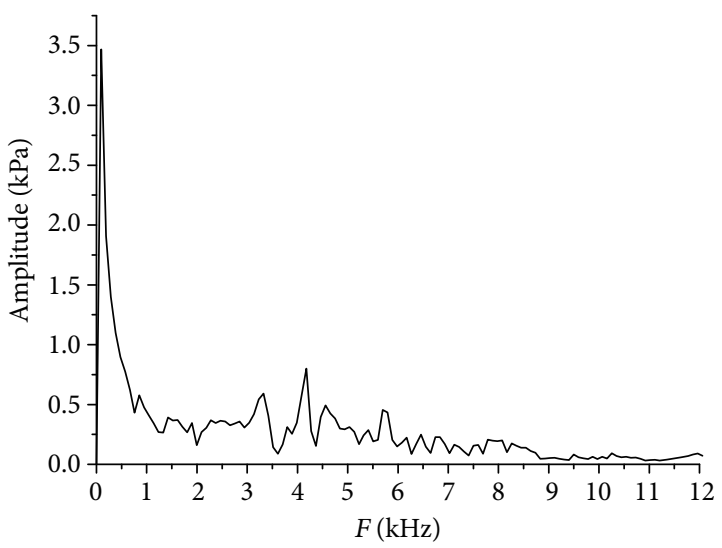

(d) $6.2 \mathrm{~cm}$

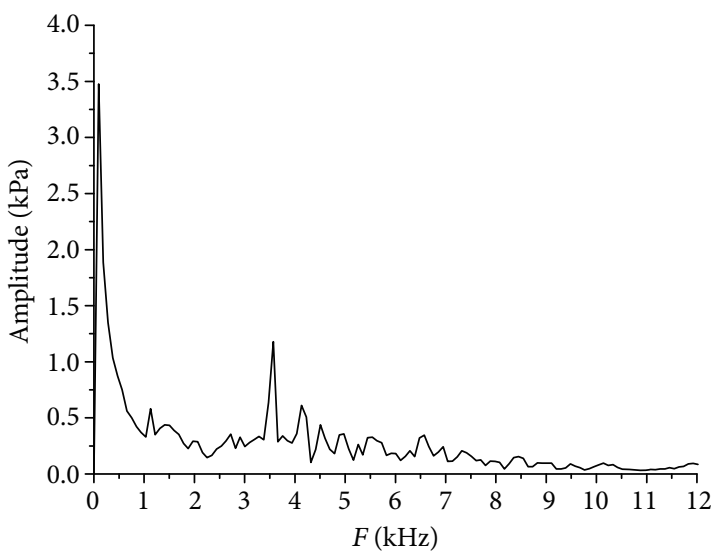

(f) $10.0 \mathrm{~cm}$

Figure 6: FFT analysis of pressure oscillations at the observation point for the Model B chamber with different injector lengths.

injector is roughly half of the eigenfrequency of the $2 \mathrm{~L} 2 \mathrm{~T}$ acoustic mode, so the injector may play as a half-wave resonator of the 2L2T mode. For the case of injector length with $4.4 \mathrm{~cm}$, the tuning frequency closes to the eigenfrequency of $1 \mathrm{~L}$ acoustic modes, and the pressure oscillation of the $1 \mathrm{~L}$ acoustic mode is inhibited completely, while it does not work for the other acoustic modes. For the case of injector length with $6.2 \mathrm{~cm}$, the tuning frequency is keeping close to the eigenfrequency of $1 \mathrm{~L}$ acoustic modes much more, and the pressure oscillation of $1 \mathrm{~L}$ acoustic modes is still repressed completely, while the pressure oscillation of $2 \mathrm{~T}$ also suppresses significantly. The peak value of the $2 \mathrm{~T}$ acoustic mode is very small enough that its half-power bandwidth cannot be read, so the damping factor for this mode is not available in Table 4. However, there is a new peak at the frequency of $3323 \mathrm{~Hz}$ for this case. So, the energy of pressure oscillation of the $2 \mathrm{~T}$ acoustic mode may transfer to this new-frequency mode which cannot be identified. For the case of injector length with $7.8 \mathrm{~cm}$, it still restrains the $1 \mathrm{~L}$ acoustic mode completely, and it is also effective for suppression of the 


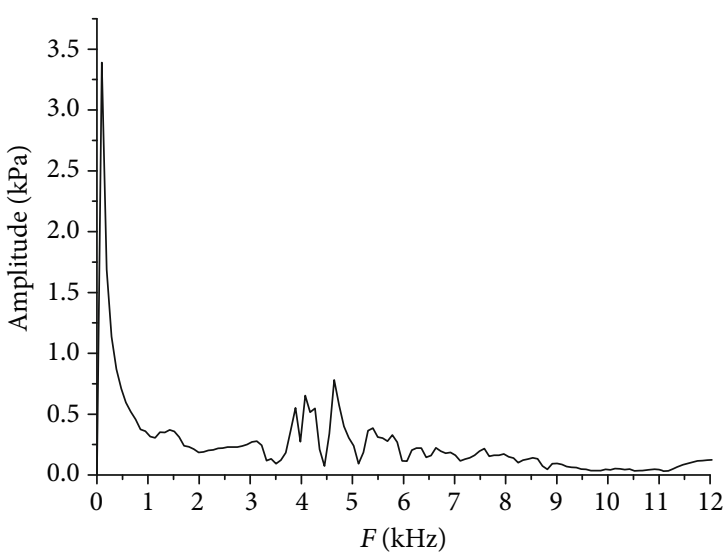

(a) $2.2 \mathrm{~cm}$

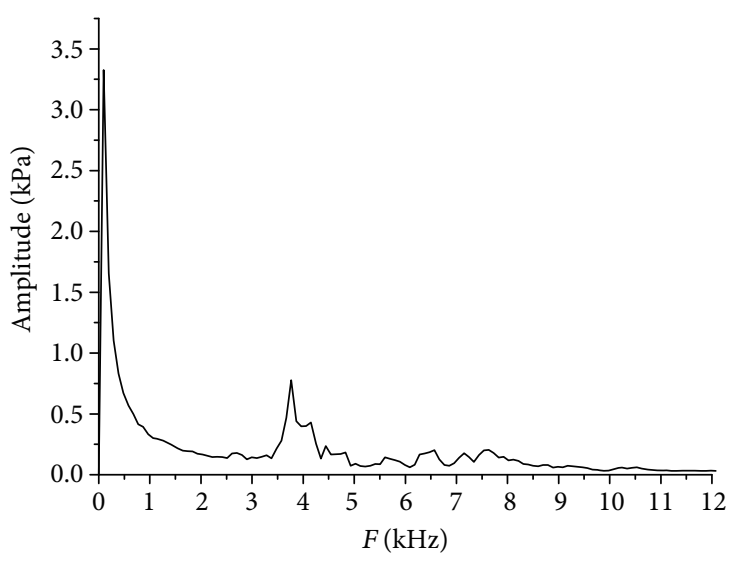

(c) $4.4 \mathrm{~cm}$

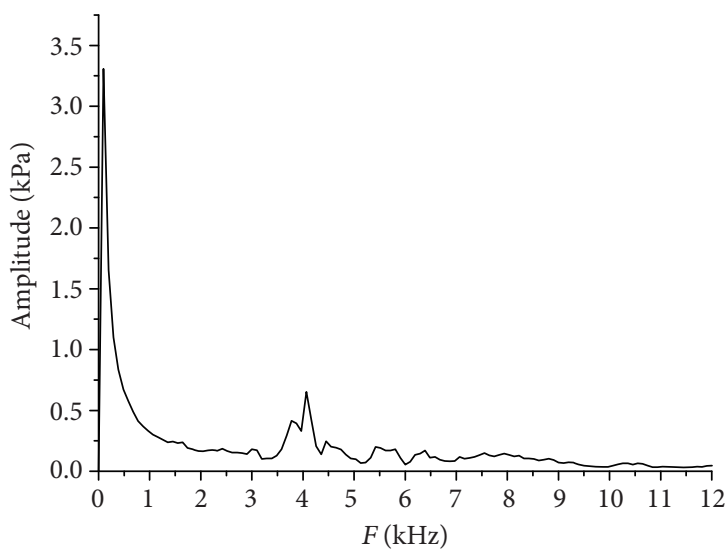

(e) $7.8 \mathrm{~cm}$

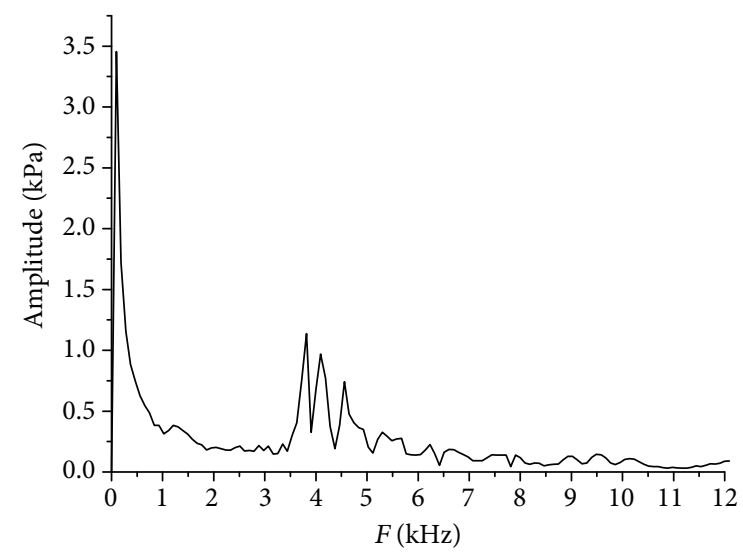

(b) $3.2 \mathrm{~cm}$

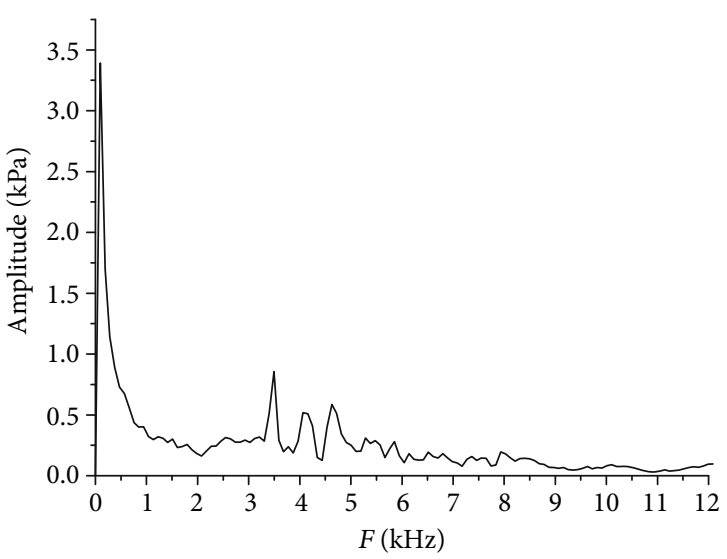

(d) $6.2 \mathrm{~cm}$

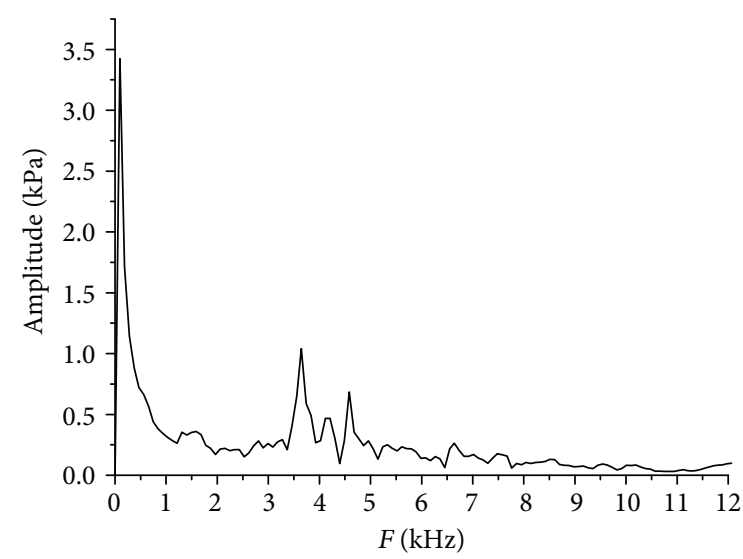

(f) $10.0 \mathrm{~cm}$

Figure 7: FFT analysis of pressure oscillations at the observation point for the Model C chamber with different injector lengths.

$2 \mathrm{~T}$ acoustic mode. The pressure oscillation of the $2 \mathrm{~L} 2 \mathrm{~T}$ acoustic mode also disappears for this case. For the case of injector length with $10 \mathrm{~cm}$, it has little effect on suppression of $1 \mathrm{~L}$ and $2 \mathrm{~T}$ acoustic modes for the tuning frequency of such injector is much less than the eigenfrequency of the acoustic modes.

For the $1 \mathrm{~L} 2 \mathrm{~T}$ and $2 \mathrm{~L} 2 \mathrm{~T}$ acoustic modes, their eigenfrequencies are larger or much larger than the tuning frequency, so suppression of injectors on these two acoustic modes has not been observed clearly. Compared with the case without an injector, the power amplitudes of these two acoustic modes increase in most cases indeed due to the redistribution of pressure oscillation energy among these acoustic modes through the injectors. In general, for the Model B chamber, injectors with length from $6.2 \mathrm{~cm}$ to $7.8 \mathrm{~cm}$ are good at suppression of pressure oscillation. The $1 \mathrm{~L}$ acoustic mode is inhibited completely for injectors are acting as quarterwave resonators, and $2 \mathrm{~T}$ is suppressed largely due to redistribution of pressure-oscillation energy through these injectors, while power amplitudes of $1 \mathrm{~L} 2 \mathrm{~T}$ and $2 \mathrm{~L} 2 \mathrm{~T}$ acoustic modes 
TABLE 5: Eigenfrequency, power amplitude, and damping rate of acoustic modes in the Model C chamber.

\begin{tabular}{|c|c|c|c|c|c|c|c|c|}
\hline & \multicolumn{7}{|c|}{ Length of the injector $(\mathrm{cm})$} \\
\hline & & 0 & 2.2 & 3.2 & 4.4 & 6.2 & 7.8 & 10 \\
\hline \multicolumn{2}{|c|}{ Resonator's tuning frequency $(\mathrm{Hz})$} & - & 3750 & 2578 & 1875 & 1330 & 1057 & 825 \\
\hline \multirow{3}{*}{$1 \mathrm{~L}$ acoustic mode } & Eigenfrequency $(\mathrm{Hz})$ & 985 & - & - & - & - & - & - \\
\hline & Amplitude (kPa) & 0.76 & - & - & - & - & - & - \\
\hline & Damping factor & $11.8 \%$ & - & - & - & - & - & - \\
\hline \multirow{3}{*}{$2 \mathrm{~T}$ acoustic mode } & Eigenfrequency $(\mathrm{Hz})$ & 3771 & 3886 & 3813 & 3756 & 3777 & 3771 & 3648 \\
\hline & Amplitude (kPa) & 1.17 & 0.55 & 1.13 & 0.78 & 0.24 & 0.41 & 1.0 \\
\hline & Damping factor & $3.4 \%$ & $4.4 \%$ & $2.2 \%$ & $4.2 \%$ & - & $3.8 \%$ & $3.6 \%$ \\
\hline \multirow{3}{*}{ 1L2T acoustic mode } & Eigenfrequency $(\mathrm{Hz})$ & 3961 & 4076 & 4092 & 4147 & 4060 & 4062 & 4160 \\
\hline & Amplitude (kPa) & 0.58 & 0.65 & 0.97 & 0.42 & 0.52 & 0.66 & 0.47 \\
\hline & Damping factor & $8.2 \%$ & $2.5 \%$ & $4.3 \%$ & $4.0 \%$ & $6.6 \%$ & $2.5 \%$ & $3.5 \%$ \\
\hline \multirow{3}{*}{ 2L2T acoustic mode } & Eigenfrequency $(\mathrm{Hz})$ & 4461 & 4644 & 4558 & - & 4627 & - & 4583 \\
\hline & Amplitude (kPa) & 0.31 & 0.78 & 0.74 & - & 0.58 & - & 0.68 \\
\hline & Damping factor & $3.2 \%$ & $2.0 \%$ & $2.5 \%$ & - & $3.3 \%$ & - & $2.1 \%$ \\
\hline \multirow{3}{*}{ Unidentified frequency peaks } & Eigenfrequency $(\mathrm{Hz})$ & - & - & - & - & 3494 & - & - \\
\hline & Amplitude (kPa) & - & - & - & - & 0.85 & - & - \\
\hline & Damping factor & - & - & - & - & $2.5 \%$ & - & - \\
\hline
\end{tabular}

increase not dramatically. $1 \mathrm{~L}$ and $2 \mathrm{~T}$ acoustic modes, which are mostly concerned by engineering, are significantly controlled by coaxial injectors.

So, such a coaxial injector with one open end and another half-open end still can work as a quarter-wave resonator. For multimode pressure oscillation, it is better for tuning frequency of the injector closing to the lower eigenfrequency acoustic mode, which will be effective for suppression of these multiacoustic modes simultaneously.

3.4. Acoustic Properties of Model C Chamber. After imposing a constant volume bomb in the Model C chamber, FFT analysis of pressure oscillations at the observation point for cases with different injector lengths is shown in Figure 7. The peak frequencies that appeared in Figure 7 are also identified by the theoretical eigenfrequencies of acoustic modes for the Model A chamber. There are three peak frequencies among these results for the Model $\mathrm{C}$ chamber; they are located between $3459 \mathrm{~Hz}$ and $3886 \mathrm{~Hz}, 4060 \mathrm{~Hz}$ and $4160 \mathrm{~Hz}$, and $4558 \mathrm{~Hz}$ and $4644 \mathrm{~Hz}$, respectively. Based on the theoretical eigenfrequencies of acoustic modes for the Model A chamber, these peak frequencies are therefore identified as eigenfrequencies of $2 \mathrm{~T}, 1 \mathrm{~L} 2 \mathrm{~T}$, and $2 \mathrm{~L} 2 \mathrm{~T}$ acoustic modes, respectively. So, the excited acoustic modes in the Model C chamber by the same numerical constant volume bomb are those of 2T, 1L2T, and 2L2T modes. Compared with those in Model A and B chambers, there is no new acoustic mode that appeared in the Model $\mathrm{C}$ chamber. So, the acoustic modes excited by the same stimulated source do not change for the case with or without injectors as well as with or without the plenum chamber. It still mainly depends on the chamber configuration for the observed acoustic-mode pressure oscillation propagation in the chamber.

The eigenfrequencies, power amplitudes, and damping rate of $2 \mathrm{~T}, 1 \mathrm{~L} 2 \mathrm{~T}$, and $2 \mathrm{~L} 2 \mathrm{~T}$ acoustic modes observed through
Figure 7 in the Model $\mathrm{C}$ chamber are listed in Table 5. The eigenfrequencies of each acoustic mode for the Model $\mathrm{C}$ chamber are close to those of the corresponding acoustic mode for the Model B chamber. So, with or without the plenum chamber does not have a significant effect on the eigenfrequencies of the corresponding acoustic modes. Compared with cases in the Model B chamber, the pressure oscillation of the $1 \mathrm{~L}$ acoustic mode is completely suppressed for all cases with different injector lengths when just the plenum chamber is considered in the Model C chamber. So, it can be concluded that the plenum chamber is very effective for suppression of $1 \mathrm{~L}$ acoustic pressure oscillation. In general, the damping rate of $2 \mathrm{~T}, 1 \mathrm{~L} 2 \mathrm{~T}$, and $2 \mathrm{~L} 2 \mathrm{~T}$ acoustic modes is a little larger for cases in the Model $\mathrm{C}$ chamber than those in the Model B chamber; it means that the plenum chamber provides an extra decay for pressure oscillation of these acoustic modes, but such decay is not significant. However, the amplitudes of 2T and 1L2T acoustic modes for cases with the plenum chamber are smaller significantly than those without the plenum chamber. So, the plenum chamber decreases the amplitudes of the acoustic modes mainly by absorbing the pressure-oscillation energy of the combustion chamber, not just by extra decay of the propagation pressure wave. Amplitudes of $2 \mathrm{~L} 2 \mathrm{~T}$ acoustic modes for cases in the Model C chamber are a little larger than those in the Model $\mathrm{B}$ chamber; it may be induced by redistribution of pressureoscillation energy among these acoustic modes through injectors. The effects of injector length on the 2T acoustic pressure oscillation for the Model $\mathrm{C}$ chamber are like those for the Model B chamber. The tuning frequency of the $2.2 \mathrm{~cm}$ length injector is very close to eigenfrequency of the $2 \mathrm{~T}$ acoustic mode; it can be found that the amplitudes of the $2 \mathrm{~T}$ acoustic mode decrease significantly as compared with the cases without injectors. From then on, the amplitudes of the $2 \mathrm{~T}$ acoustic mode increase with the length of injectors. It 


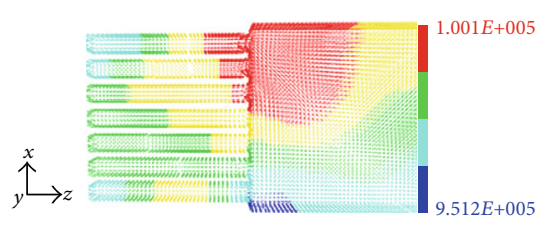

(a) Pressure wave propagating into the injectors

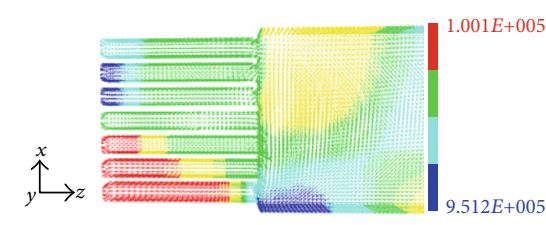

(b) Pressure wave propagating into the chamber where pressure is in the lowest value

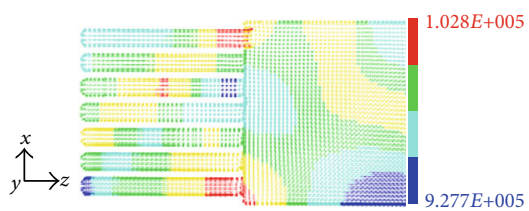

(c) Pressure wave propagating into the chamber where pressure is in the middle value

FIGURE 8: Instantaneous velocity vector and pressure fields in injectors and chamber.

means that the injectors still can work as a quarter-wave resonator with the plenum chamber.

\subsection{Effects of Injectors and Plenum Chamber on the Pressure} Oscillation. Figure 8 shows the instantaneous velocity vector and pressure fields in the chamber and injector. As shown in Figure $8(\mathrm{a})$, pressure waves propagate into the injectors when pressures at the chamber zone adjacent to injectors are in high value. It means that some energies of pressure oscillation are absorbed by injectors from their peak. As shown in Figure $8(\mathrm{~b})$, the pressure waves propagated into injectors from the chamber reflect at the end of the injectors and go towards the chamber. When it arrives at the exit of the injector, the pressure at the chamber zone adjacent to the injectors is just in its lowest value. So, the absorbed energy from the peak of pressure oscillation is added to a trough of pressure oscillation. In this way, which is called Cutting down the pressure Peak and Raising up the pressure Trough (CPRT), the power amplitudes of the acoustic mode, by which eigenfrequency is close to the tuning frequency of injectors, are suppressed significantly as shown in Model B and C chambers. As shown in Figure 8(c), when the pressure wave in injectors arrives at the exit of injectors, the pressure at the chamber zone adjacent to the injectors is in the middle value. It means that the pressure at this zone is controlled by other acoustic modes which are different from the acoustic mode that this pressure wave is induced. So, the energy of pressure oscillation is transformed from one acoustic mode to other acoustic modes through these injectors. So, as shown in Model B and C chambers, such redistribution of pressure oscillation energy leads to an increase of power amplitudes of some acoustic modes when injectors are considered and multimode pressure oscillations present in the chamber.

Figure 9 shows the instantaneous velocity vector and pressure fields in the plenum chamber and injector. For the lower four injectors, the pressure at the injector zone adjacent to the plenum chamber is in high value; pressure waves thereby propagate from injectors to the plenum chamber. Such propagations of pressure waves are easily and frequently observed in the numerical results. The pressure waves propagated from the plenum chamber to the injector can also be observed as upper three injectors, but its ampli-

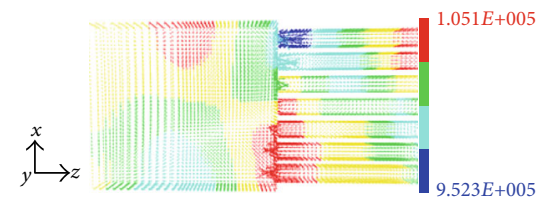

FIGURE 9: Instantaneous velocity vector and pressure fields in injectors and plenum chamber.

tude is obviously smaller than that propagated from injectors to the plenum chamber. That is the reason why the pressure oscillation of most acoustic modes can be suppressed significantly by the plenum chamber.

\section{Conclusion}

The effects of coaxial injectors and plenum chamber on the acoustic properties of the thruster chamber are numerically investigated, in which a numerical constant volume bomb is employed to excite multimode pressure oscillation. FFT analysis and half-power bandwidth method are applied to get the eigenfrequency, power amplitude, and damping rate of each excited acoustic mode. Under the same stimulated source, the excited acoustic modes are the same as $1 \mathrm{~L}, 2 \mathrm{~T}$, $1 \mathrm{~L} 1 \mathrm{~T}$, and 2L2T for chambers with and without injectors as well as with and without plenum chamber, and their eigenfrequencies and damping rates do not have obvious changes. The injectors still act as a quarter-wave resonator under multimode pressure oscillations for they are very effective to suppress acoustic-mode pressure oscillation when its eigenfrequency is close to the tuning frequency of injectors. And it is better for tuning frequency of the injector closing to the lower eigenfrequency acoustic mode, which will be effective for suppression of these multiacoustic modes simultaneously. Comparing the pressure oscillation with and without the plenum chamber, the plenum chamber can inhibit 1L acoustic-mode pressure oscillation completely and also has obvious effects on other acoustic modes. For multimode pressure oscillation, it can be found that there is a redistribution of pressure-oscillation energy among those excited acoustic modes. 


\section{Data Availability}

The data used to support the findings of this study are available from the corresponding authors upon request.

\section{Conflicts of Interest}

The authors declare that they have no conflicts of interest.

\section{Acknowledgments}

This work is supported by the key program of the National Natural Science Foundation of China under Grant No. 91841303.

\section{References}

[1] J. J. Hutt and M. Rocker, "High-frequency injection-coupled combustion instability," Progress in Astronautics and Aeronautics, vol. 169, pp. 345-356, 1995.

[2] H. Kawashima, K. KOBAYASHI, T. Tomita, and T. Kaneko, "A combustion instability phenomenon on a LOX/methane subscale combustor," in 6th AIAA/ASME/SAE/ASEE Joint Propulsion Conference \& Exhibit, Nashville, TN, 2010.

[3] R.-S. Lin, F. Bertolotti, C. Eckett, T. Hinerman, and R. Jensen, "A study of the application of a finite element model to the predictions of the injection-coupled response of a LOX post," in 46th AIAA/ASME/SAE/ASEE Joint Propulsion Conference \& Exhibit, Nashville, TN, 2010.

[4] S. Gröning, J. S. Hardi, D. Suslov, and M. Oschwald, "Injectordriven combustion instabilities in a hydrogen/oxygen rocket combustor," Journal of Propulsion and Power, vol. 32, no. 3, pp. 560-573, 2016.

[5] A. Urbano, L. Selle, G. Staffelbach et al., "Exploration of combustion instability triggering using large eddy simulation of a multiple injector liquid rocket engine," Combustion and Flame, vol. 169, pp. 129-140, 2016.

[6] J. Zhou, X. Hu, Y. Huang, and Z. Wang, "Flowrate and acoustics characteristics of coaxial swirling injector of hydrogen/oxygen rocket engine," in 32nd Joint Propulsion Conference and Exhibit, Lake Buena Vista, FL, U.S.A., 1996.

[7] J. Zhou, X. Hu, and Y. Huang, "An experimental study on acoustic characteristics of gas-liquid coaxial injector of liquid rocket engine," Journal of Propulsion Technology-Beijing-, pp. 37-41, 1996.

[8] C. H. Sohn, Y. J. Kim, Y.-M. Kim, and V. P. Pikalov, "A scaling method for combustion stability rating of coaxial gas-liquid injectors in a subscale chamber," Journal of mechanical science and technology, vol. 26, no. 11, pp. 3691-3699, 2012.

[9] C. H. Sohn, I. S. Park, S. K. Kim, and H. J. Kim, "Acoustic tuning of gas-liquid scheme injectors for acoustic damping in a combustion chamber of a liquid rocket engine," Journal of Sound and Vibration, vol. 304, no. 3-5, pp. 793-810, 2007.

[10] H. Kim and C. H. Sohn, "Experimental study of the role of gasliquid scheme injector as an acoustic resonator in a combustion chamber," Journal of Mechanical Science and Technology, vol. 20, no. 6, pp. 896-904, 2006.

[11] S. Soller, R. Wagner, H.-P. Kau, P. Martin, and C. Maeding, "Combustion stability characteristics of coax-swirl-injectors for oxygen/kerosene," in 43rd AIAA/ASME/SAE/ASEE Joint Propulsion Conference \& Exhibit, Cincinnati, OH, 2007.
[12] B. Pomeroy, C. Morgan, and W. Anderson, "Response of a gascentered swirl coaxial injector to transverse instabilities," in 47th AIAA/ASME/SAE/ASEE Joint Propulsion Conference \& Exhibit, San Diego, California, 2011.

[13] B. Pomeroy, J. Sisco, J. Eckstein, and W. Anderson, "Characterization of gas-centered swirl-coaxial injector stability in a subscale multi-element combustor," in 44th AIAA/ASME/SAE/ASEE Joint Propulsion Conference \& Exhibit, Hartford,CT, 2008.

[14] B. D. Kim and S. D. Heister, "Two-phase modeling of hydrodynamic instabilities in coaxial injectors," Journal of Propulsion and Power, vol. 20, no. 3, pp. 468-479, 2004.

[15] V. P Zhukov and M. Feil, "Numerical Simulations of the Flame of a Single Coaxial Injector," International Journal of Aerospace Engineering, vol. 2017, 11 pages, 2017.

[16] J. Jeon, M. Hong, Y. M. Han, and S. Y. Lee, "Experimental study on spray characteristics of gas-centered swirl coaxial injectors," Journal of Fluids Engineering, vol. 133, no. 12, p. 121303, 2011.

[17] W. E. Anderson and V. Yang, Eds., Liquid rocket engine combustion instability, American Institute of Aeronautics and Astronautics, 1995.

[18] J. Qin, H. Zhang, and B. Wang, "Numerical evaluation of acoustic characteristics and their damping of a thrust chamber using a constant-volume bomb model," Chinese Journal of Aeronautics, vol. 31, no. 3, pp. 470-480, 2018. 